\title{
About Generating Set of the Invariant Subalgebra of Free Restricted Lie Algebra
}

\author{
V. M. Petrogradsky ${ }^{1}$, I. A. Subbotin ${ }^{2}$ \\ ${ }^{1}$ Department of Mathematics, University of Brasilia, 70910-900 Brasilia DF, Brazil, petrogradsky@ rambler.ru \\ ${ }^{2}$ Ulyanovsk State University, Russia, 432970, Ulyanovsk, ul. L'va Tolstogo, 42, shelby888@yandex.ru \\ Suppose that $L=L(X)$ is the free Lie $\mathrm{p}$-algebra of finite rank $k$ with free generating set $X=\left\{x_{1}, \ldots, x_{k}\right\}$ on a field of positive \\ characteristic. Let $G$ is nontrivial finite group of homogeneous automorphisms $L(X)$. Our main purpose to prove that $L^{G}$ subalgebra \\ of invariants is is infinitely generated. We have more strongly result. Let $Y=\cup_{n=1}^{\infty} Y_{n}$ be homogeneous free generating set for \\ the algebra of invariants $L^{G}$, elements $Y_{n}$ are of degree $n$ relatively $X, n \geq 1$. Consider the corresponding generating function \\ $\mathscr{H}(Y, t)=\sum_{n=1}^{\infty}\left|Y_{n}\right| t^{n}$. In our case of free Lie restricted algebras, we prove, that series $\mathscr{H}(Y, t)$ has a radius of convergence \\ $1 / k$ and describe its growth at $t \rightarrow 1 / k-0$. As a result we obtain that the sequence $\left|Y_{n}\right|, n \geq 1$, has exponential growth. \\ Key words: free Lie algebras, Lie p-algebras, invariants, generating set.
}

\section{References}

1. Shirshov A. I. Subalgebras of free Lie algebra. Mat. sb., 1953, vol. 33, no. 2, pp. 441-452 (in Russian).

Gruyter Exp. Math., Berlin, Walter de Gruyter \& Co., 1992, vol. 7, 250 p.

2. Witt E. Die Unterringe der freien Lieschen Ringe. Math. Z., 1956, vol. 64, pp. 195-216.

3. Bryant R. M. On the fixed points of a finite group acting on a free Lie algebra. J. London Math. Soc. 1991, vol. 43, no. 2, pp. 215-224.

4. Petrogradsky V. M., Smirnov A. A. On invariants of modular free Lie algebras. J. Math. Sci., 2010, vol. 166, no. 6, pp. $767-772$.

5. Jacobson N. Lie algebras. New York, Interscience, 1962. 332 p.

6. Bahturin Yu. A. Identical Relations in Lie Algebras. Netherlannds, VNU Sciens Press BV, 1987, 309 p.

7. Petrogradsky V. M. On Witt's formula and invariants for free Lie superalgebras. Formal power series and algebraic combinatorics (Moscow 2000), Springer, 2000, pp. 543-551.

8. Bahturin Yu. A., Mikhalev A. A., Petrogradsky V. M., Zaicev M. V. Infinite-dimensional Lie superalgebras. de 9. Petrogradsky V. M. Witt's formula for restricted Lie algebras. Adv. Appl. Math., 2003, vol. 30, pp. 219-227.

10. Petrogradsky V. M. Asymptotic problems in algebraic structures. Limit of graphs in group theory and computer science. ed. G. Arzhantseva, A. Valette, Lausanne, EPFL Press, 2009, pp. 77-108.

11. Markushevich A. L., Markushevich L. A. Vvedenie $v$ teoriiu analiticheskikh funktsii [Introduction to the theory of analytic functions]. Moscow, Prosveshchenie, 1977, 320 p. (in Russian).

12. Petrogradsky V. M. On invariants of the action of a finite group on a free lie algebra. Sib. Math. J., 2000, vol. 41 , no. $4,763-770$.

13. Petrogradsky V. M. Characters and invariants for free Lie superalgebras. St. Petersburg Math. J., 2002, vol. 13, no. 1, pp. 107-122.

\section{УДК 517.54}

\section{ИНТЕГРАЛЫ УРАВНЕНИЯ ЛЕВНЕРА СО СТЕПЕННОЙ УПРАВЛЯЮЩЕЙ ФУНКЦИЕЙ}

\section{Д. В. Прохоров ${ }^{1}$, К. А. Самсонова ${ }^{2}$}

\begin{abstract}
${ }^{1}$ Просрессор, заведующий кафредрой математического анализа, Саратовский государственный университет им. Н. Г. Чернышевского, ProkhorovDV@info.sgu.ru

${ }^{2}$ Аспирант касредры математического анализа, Саратовский государственный университет им. Н. Г. Чернышевского, kris_ruzhik@mail.ru

Рассматривается качественное локальное поведение траекторий обыкновенного дисрференциального уравнения Левнера с управляющей функцией, обратной к степенной фрункции, с целым показателем степени. Выделены все особые точки и соответствующие им сингулярные решения. Показано, что эта управляющая фрункция порождает решения уравнения Левнера, которые представляют собой отображения полуплоскости с гладким разрезом на верхнюю полуплоскость. Найдено асимптотическое соотношение между гармоническими мерами сторон разреза.
\end{abstract}

Ключевые слова: уравнение Левнера, гармоническая мера, сингулярное решение, управляющая фуннция, $C^{1}$-кривая. 


\section{ВВЕДЕНИЕ}

На протяжении многих лет дифференциальное уравнение Левнера [1] служило мощным средством изучения свойств однолистных функций в единичном круге. Обнаруженные связи теории Левнера со многими разделами математики объясняют растущий интерес к ней в современных исследованиях (см. например [2]). Уравнение Левнера для верхней полуплоскости $\mathbb{H}$ появилось значительно позднее (см. например [3, с. 229]) и стало особенно популярным в последние десятилетия. Рассмотрим его подробнее. Пусть функция $w=f(z, t), z \in \mathbb{H}, t \geq 0$, имеющая в окрестности бесконечно удаленной точки представление

$$
f(z, t)=z+\frac{2 t}{z}+O\left(\frac{1}{z^{2}}\right), \quad z \rightarrow \infty,
$$

отображает $\mathbb{H} \backslash K_{t}, K_{t} \subset \mathbb{H}$, на $\mathbb{H}$ и является решением обыкновенного дифференциального уравнения Левнера для верхней полуплоскости:

$$
\frac{d f(z, t)}{d t}=\frac{2}{f(z, t)-\lambda(t)}, \quad f(z, 0)=z, \quad z \in \mathbb{H},
$$

с непрерывной вещественной управляющей функцией $\lambda(t)$.

Конформные отображения $f(z, t)$ допускают непрерывное продолжение на множество всех точек $z \in \mathbb{R}$, не принадлежащих замыканию множества $K_{t}$. Продолженные таким образом отображения $f(z, t)$ удовлетворяют уравнению (2). Давняя задача (см. например [4]) заключается в том, чтобы определить в терминах $\lambda$ случаи, когда $K_{t}$ оказывается жордановой дугой $\gamma(\tau), \tau \geq 0$, с начальной точкой $\gamma(0) \in \mathbb{R}$. В этом случае $\gamma(t)$ является разрезом полуплоскости $\mathbb{H}$, а $f(z, t)$ непрерывно продолжается на множество достижимых граничных точек на обеих сторонах разреза $\gamma(t)$ :

$$
\lambda(t)=f(\gamma(t), t), \quad \gamma(t)=f^{-1}(\lambda(t), t) .
$$

Точки $\gamma(t)$ рассматриваются как носители простых концов, различных на разных сторонах дуги. Известны примеры управляющих функций в уравнении Левнера, которые генерируют отображения $\mathbb{H} \backslash K_{t} \rightarrow \mathbb{H}$ с круговыми двуугольниками $K_{t}$. Для классического уравнения Левнера подобный пример построен Куфаревым [5]. Его аналог (2) для верхней полуплоскости возникает при $\lambda(t)=3 \sqrt{2} \sqrt{1-t}$, детальное описание дано в $[4,6]$.

Линейным преобразованиям управляющей функции $\lambda(t)$ соответствуют определенные преобразования решений уравнения (2). Поэтому без ограничения общности можно считать, что $\lambda(0)=0$. Геометрическое описание критических траекторий для интегралов уравнения (2) приводится в ряде статей $[4,6,7]$, как в случае интегрирования уравнения в квадратурах, так и для управляющих функций из разных функциональных классов, например класса Lip(1/2).

В настоящей статье исследуется качественное асимптотическое поведение решений дифференциального уравнения (2), генерируемых управлениями, обратными к степенной функции с натуральной степенью. Управляющая функция $\lambda(t)=\sqrt[N]{t}, N \in \mathbb{N}, N \geq 3$, выбрана как типичный представитель класса $\operatorname{Lip}(1 / N)$. Основной результат содержится в следующей теореме.

Теорема 1. Пусть $f(z, t)$ является решением дифференциального уравнения:

$$
\frac{d f(z, t)}{d t}=\frac{2}{f(z, t)-\sqrt[N]{t}}, \quad f(z, 0)=z, \quad \operatorname{Im} z \geq 0, \quad N \in \mathbb{N}, \quad N \geq 3 .
$$

Тогда для достаточно мальх $t>0 f(\cdot, t)$ отображает область $D(t)=\mathbb{H} \backslash \gamma(t)$ на $\mathbb{H}$, где $\gamma(t)$ является $C^{1}$-кривой, лежащей в $\mathbb{H}$, за исключением, быть может, точки $\gamma(0)=0$.

В силу результатов статьи [8], что если $N \geq 3$, то разрез $\gamma(t)$ в теореме 1 не может находиться в угле Штольца с вершиной в точке $z=0$. В [9] показано, что круговой разрез $\gamma(t)$, касающийся вещественной оси $\mathbb{R}$ в точке $z=0$, генерируется управляющей функцией $\lambda(t) \in \operatorname{Lip}(1 / 3)$ дифференциального уравнения (2). 
Во второй части статьи приводятся предварительные сведения об особых точках и соответствующих сингулярных решениях дифференциального уравнения и их представлении. В третьей части даются вспомогательные результаты, характеризующие поведение сингулярных решений. Четвертая часть содержит описание важных траекторий, порожденных уравнением Левнера, и доказательство основной теоремы 1. В пятой, заключительной, части доказана теорема 2 об асимптотическом поведении отношения гармонических мер двух сторон разреза, генерируемого управляющей функцией уравнения Левнера.

\section{1. ПРЕДВАРИТЕЛЬНЫЕ СВЕДЕНИЯ}

Сделаем замену переменных $t \rightarrow \tau^{N}, g(z, \tau):=f\left(z, \tau^{N}\right)$. Тогда уравнение (3) примет вид

$$
\frac{d g(z, \tau)}{d \tau}=\frac{2 N \tau^{N-1}}{g(z, \tau)-\tau}, \quad g(z, 0)=z, \quad \operatorname{Im} z \geq 0
$$

Если $z \neq 0$, то $g(z, 0) \neq 0$ и существует регулярное решение $g(z, \tau)$ уравнения (4), голоморфное относительно $\tau$ при достаточно малых $|\tau|$, единственное для каждого $z \neq 0$. Сингулярные решения уравнения (4) не удовлетворяют условиям единственности. Каждая точка $\left(g\left(z_{0}, \tau_{0}\right), \tau_{0}\right)$ такая, что $g\left(z_{0}, \tau_{0}\right)=\tau_{0}$, является сингулярной точкой для уравнения $(4)$. Если $\tau_{0} \neq 0$, то точка $\left(g\left(z_{0}, \tau_{0}\right), \tau_{0}\right)$ называется алгебраической критической точкой решения $g(z, \tau)$. В этом случае соответствующие сингулярные решения уравнения (4) разложимы в ряды по степеням $\left(\tau-\tau_{0}\right)^{1 / m}, m \in \mathbb{N}$, в окрестности точки $\tau=\tau_{0}$ (см. [10, с. 129]).

Точка $\left(g\left(z_{0}, \tau_{0}\right), \tau_{0}\right)=(0,0)$ является единственной сингулярной точкой неопределенного характера, для которой числитель и знаменатель в правой части уравнения (4) обращаются в нуль одновременно [10, с. 130].

Поведение всех решений уравнения (4) описывается теоремой Пуанкаре-Бендиксона [10, с. 138; $11 ; 12]$. Две интегральные кривые дифференциального уравнения (4) пересекаются только в особой точке $(0,0)$. Интегральная кривая уравнения (4) может иметь кратные точки только в точке $(0,0)$. Бендиксон (I. Bendixson) [12] установил, что вещественные интегральные кривые имеют конечные точки в узлах и фокусах и имеют продолжение через седловые точки. Теорема Бендиксона [12] описывает все три возможных случая поведения траекторий для уравнения (4) в окрестности точки $(0,0)$ : (а) интегральная кривая замкнута, то есть является циклом; (б) интегральная кривая представляет собой спираль, которая стремится к циклу асимптотически; (c) интегральная кривая имеет конечную точку $(0,0)$.

Напомним случаи интегрируемости в квадратурах дифференциального уравнения Левнера (2), приведенные в [6] и соответствующие управляющим функциям $\lambda(t)=c \sqrt{t}$ и $\lambda(t)=c \sqrt{1-t}$. В первом из них, после замены переменной $t \rightarrow \tau^{2}$, особая точка $(0,0)$ становится седловой по классификации Пуанкаре [11], а во втором замена переменной $t \rightarrow 1-\tau^{2}$ приводит к фокусу в особой точке $(0,0)$.

Будем искать решения уравнения (4), которые бесконечно дифференцируемы относительно действительной переменной $\tau$ (см. [10, с. 129; 13, с. 13]). Рекуррентные оценки тейлоровских коэффициентов позволяют отыскать сингулярные решения при условии, что получившийся ряд будет иметь положительный радиус сходимости [14, с. 101]. Пусть

$$
g_{s}(0, \tau)=\sum_{n=1}^{\infty} a_{n} \tau^{n}
$$

представляет формальное степенное разложение для сингулярного решения уравнения (4). Такое разложение не единственно, оно зависит от пути, по которому $z$ приближается к $0, z \notin K_{t}$. Подставляя (5) в (4), получаем:

$$
\sum_{n=1}^{\infty} n a_{n} \tau^{n-1}\left(\sum_{n=1}^{\infty} a_{n} \tau^{n}-\tau\right)=2 N \tau^{N-1}
$$


Приравниваем коэффициенты при одинаковых степенях в обеих частях (6) и получаем систему уравнений

$$
\begin{gathered}
a_{1}\left(a_{1}-1\right)=0 \\
\sum_{k=1}^{l} k a_{k} a_{l-k+1}-l a_{l}=0, \quad l=2, \ldots, \quad l \neq N-1, \\
\sum_{k=1}^{N-1} k a_{k} a_{N-k}-(N-1) a_{N-1}=2 N
\end{gathered}
$$

Первое уравнение системы (7) предполагает два возможных значения $a_{1}=1$ и $a_{1}=0$ для двух сингулярных решений $g^{+}(0, \tau)$ и $g^{-}(0, \tau)$. В обоих случаях уравнение $(6)$ предполагает рекуррентные формулы для коэффициентов $a_{l}^{+}$и $a_{l}^{-}$функций $g^{+}(0, \tau)$ и $g^{-}(0, \tau)$ соответственно:

$$
\begin{gathered}
a_{1}^{+}=1, \quad a_{2}^{+}=\ldots=a_{N-2}^{+}=0, \quad a_{N-1}^{+}=2 N, \quad a_{l}^{+}=-\sum_{k=2}^{l-1} k a_{k}^{+} a_{l-k+1}^{+}, \quad l \geq N, \\
a_{1}^{-}=\ldots=a_{N-2}^{-}=0, \quad a_{N-1}^{-}=-\frac{2 N}{N-1}, \quad a_{l}^{-}=\frac{1}{l} \sum_{k=2}^{l-1} k a_{k}^{-} a_{l-k+1}^{-}, \quad l \geq N .
\end{gathered}
$$

Покажем, что ряд $\sum_{n=1}^{\infty} a_{n}^{+} \tau^{n}$, формально представляющий $g^{+}(0, \tau)$, расходится для всех $\tau \neq 0$.

Лемма 1. Если $s \neq(N-2) m+1, m=0,1, \ldots$, mо $a_{s}^{+}=0$. Существуют $c_{1 N}>0 u c_{2 N}>0$ maкие, что для всех $l=(N-2) m+1, m=0,1, \ldots$, выполняются неравенства

$$
c_{1 N}^{m} m ! \leq\left|a_{l}^{+}\right| \leq c_{2 N}^{l-1} l^{l-3} .
$$

Доказательство. Докажем, что $a_{s}^{+}=0$ для $s \neq(N-2) m+1, m=0,1, \ldots$ Из формулы (8) следует, что $a_{s}^{+}=0$ для начальных значений $s=2, \ldots, N-2$. Предположим, что $a_{s}^{+}=0$ для всех $s \neq(N-2) q+1, q=0,1, \ldots, m$, и докажем, что $a_{s}^{+}=0$ для $(N-2) m+2 \leq s \leq(N-2)(m+1)$. Согласно (8)

$$
a_{s}^{+}=-\sum_{k=2}^{s-1} k a_{k}^{+} a_{s-k+1}^{+}
$$

Если $k=(N-2) q+1, q=0,1, \ldots, m$, то $s-k$ не может быть кратно $N-2$. Поэтому по предположению индукции $a_{s-k+1}^{+}=0$ в формуле $(11)$ и $a_{s}^{+}=0$, что доказывает индуктивное утверждение.

Далее докажем, что $a_{l}^{+}=(-1)^{q+1}\left|a_{l}^{+}\right|$, где $l=(N-2) q+1, q=1,2, \ldots$ Из формулы (8) следует, что $a_{N-1}^{+}=2 N>0$ для $q=1$. Предположим, что $a_{l}^{+}=(-1)^{q+1}\left|a_{l}^{+}\right|$для всех $l=(N-2) q+1$, $q=1, \ldots, s-1$, и докажем, что $a_{l}^{+}=(-1)^{s+1}\left|a_{l}^{+}\right|$для $l=(N-2) s+1$. Согласно (8) и предположению индукции получаем:

$$
\begin{gathered}
a_{(N-2) s+1}^{+}=-\sum_{p=1}^{s-1}((N-2) p+1) a_{(N-2) p+1}^{+} a_{(N-2)(s-p)+1}^{+}= \\
=(-1)^{s+1} \sum_{p=1}^{s-1}((N-2) p+1)\left|a_{(N-2) p+1}^{+}\right|\left|a_{(N-2)(s-p)+1}^{+}\right|=(-1)^{s+1}\left|a_{(N-2) s+1}^{+}\right|,
\end{gathered}
$$

что доказывает индуктивное утверждение.

Перейдем к доказательству нижней оценки в неравенстве (10). Для $a_{N-1}^{+}=2 N$ нижняя оценка (10) выполняется. Предположим, что эта оценка выполняется для $a_{l}^{+}$, где $l=(N-2) s+1$, $s=1, \ldots, m-1$, и докажем, что нижняя оценка (10) справедлива для $l=(N-2) m+1$. Действительно,

$$
\left|a_{(N-2) m+1}^{+}\right|=\sum_{p=1}^{m-1}((N-2) p+1)\left|a_{(N-2) p+1}^{+}\right|\left|a_{(N-2)(m-p)+1}^{+}\right| \geq
$$




$$
\geq \sum_{p=1}^{m-1}((N-2) p+1) c_{1 N}^{p} p ! c_{1 N}^{m-p}(m-p) ! \geq c_{1 N}^{m} \sum_{p=1}^{m-1}(p+1) !(m-p) ! \geq c_{1 N}^{m} m !
$$

что индуктивно доказывает нижнюю оценку (10).

Теперь получим верхнюю оценку в неравенстве (10). Существует $c_{2 N}$ такое, что справедлива оценка $\left|a_{n}^{+}\right| \leq c_{2 N}^{n-1} n^{n-3}$ для $n=1,2,3$. Предположим, что утверждение верно для $a_{k}^{+}$, где $k=1, \ldots, n-1$, $n \geq 4$, и докажем, что верхняя оценка (10) справедлива для $k=n$. Действительно,

$$
\begin{gathered}
\left|a_{n}^{+}\right|=\sum_{k=2}^{n-1} k\left|a_{k}^{+}\right|\left|a_{n-k+1}^{+}\right| \leq c_{2 N}^{n-1} \sum_{k=2}^{n-1} k^{k-2}(n-k+1)^{n-k-2}= \\
=c_{2 N}^{n-1} n^{n-4}\left((1-1 / n)^{n-4}+\sum_{k=3}^{n-3}(k / n)^{k-2}\left(\frac{n-k+1}{n}\right)^{n-k-2}+(1-2 / n)^{n-4}+\frac{(1-1 / n)^{n-2}}{2}\right) .
\end{gathered}
$$

Для $n \geq 4$ справедливы неравенства

$$
(1-1 / n)^{n-4} \leq 1, \quad(1-2 / n)^{n-4} \leq 1, \quad(1-1 / n)^{n-3} \leq 3 / 4 .
$$

Кроме того, для $3 \leq k \leq n-3, n \geq 6$, очевидно, справедливы неравенства

$$
(k / n)^{k-2}\left(\frac{n-k+1}{n}\right)^{n-k-2}<1 / 2 .
$$

Подставляем эти неравенства в (12) и приходим к неравенству

$$
\left|a_{n}^{+}\right|<c_{2 N}^{n-1} n^{n-4}\left(1+\sum_{k=3}^{n-3} \frac{1}{2}+1+\frac{3 n}{8}\right)<c_{2 N}^{n-1} n^{n-3}
$$

которое доказывает верхнюю оценку (10) и завершает доказательство леммы 1.

Лемма 2. Если $s \neq(N-2) m+1, m=1,2, \ldots$, mо $a_{s}^{-}=0$. Для всех $l=(N-2) m+1, m=1,2, \ldots$, выполняются неравенства

$$
\left|a_{l}^{-}\right| \leq c_{2 N}^{l-1} l^{l-3}
$$

Доказательство. Докажем, что $a_{s}^{-}=0$ для $s \neq l$. Из рекуррентной формулы (9) следует, что $a_{s}^{-}=0$ для начальных значений $s=1,2, \ldots, N-2$. Предположим, что $a_{s}^{-}=0$ для всех $s \neq(N-2) q+1$, $q=1, \ldots, m$, и докажем, что $a_{s}^{-}=0$ для $(N-2) m+2 \leq s \leq(N-2)(m+1)$. Согласно (9)

$$
a_{s}^{-}=\frac{1}{s} \sum_{k=2}^{s-1} k a_{k}^{-} a_{s-k+1}^{-}
$$

Если $k=(N-2) q+1, q=0,1, \ldots, m$, то $s-k$ не может быть кратно $N-2$. Поэтому по предположению индукции $a_{s-k+1}^{-}=0$ в формуле (14) и $a_{s}^{-}=0$, что доказывает индуктивное предположение.

Доказательство того, что $a_{l}^{-}=(-1)^{q}\left|a_{l}^{-}\right|$, где $l=(N-2) q+1, q=1,2, \ldots$, проводится аналогично тому, как в лемме 1 было показано, что $a_{l}^{+}=(-1)^{q+1}\left|a_{l}^{+}\right|$.

Доказательство неравенства (13) следует из леммы 1. Действительно, $a_{1}^{-}<a_{1}^{+},\left|a_{N-1}^{-}\right|<\left|a_{N-1}^{+}\right|$. Из формул (8) и (9) индуктивно следует, что для всех $l=(N-2) m+1$ справедливы неравенства $\left|a_{l}^{-}\right|<\left|a_{l}^{+}\right|$, что вместе с утверждением леммы 1 доказывает лемму 2.

Из нижней оценки (10) следует расходимость ряда $\sum_{l=1}^{\infty} a_{l}^{+} \tau^{l}$ при $\tau \neq 0$. Следовательно, уравнение (4) не имеет голоморфного решения (5) в окрестности точки $\tau_{0}=0$ с условием $a_{1}^{+}=1$. Известный метод Бореля $[14$, с. $107 ; 15]$, позволяет суммировать расходящийся ряд $\sum_{l=1}^{\infty} a_{l}^{+} \tau^{l}$. Согласно лемме 1 ряд

$$
G(\tau)=\sum_{n=1}^{\infty} \frac{a_{n}^{+}}{n !} \tau^{n}
$$


сходится при $|\tau|<R_{0}, R_{0}>0$. Сумма Бореля

$$
h(\tau)=\int_{0}^{\infty} e^{-x} G(\tau x) d x
$$

определяет бесконечно дифференцируемую функцию $h(\tau), h^{(n)}(0)=a_{n}^{+}, n \geq 1$, которая является решением уравнения (4). Такой же подход можно применить и к ряду $\sum_{n=1}^{\infty} a_{n}^{-} \tau^{n}$.

В этих случаях решения $g^{+}(0, \tau), g^{-}(0, \tau)$ уравнения (4) с начальной особой точкой $(0,0)$ удовлетворяют асимптотическим соотношениям:

$$
g^{+}(0, \tau)=\sum_{k=1}^{n} a_{k}^{+} \tau^{k}+0\left(\tau^{n}\right), \quad g^{-}(0, \tau)=\sum_{k=1}^{n} a_{k}^{-} \tau^{k}+0\left(\tau^{n}\right), \quad \tau \rightarrow 0,
$$

для всех $n \geq 2$.

Пусть $f_{1}(0, t):=g^{+}(0, \sqrt[N]{t}), f_{2}(0, t):=g^{-}(0, \sqrt[N]{t})$. Так как $f_{1}(0, t)=\sqrt[N]{t}+2 N \sqrt[N]{t^{N-1}}+O\left(\sqrt[N]{t^{N-1}}\right)$ и $f_{2}(0, t)=-\frac{2 N}{N-1} \sqrt[N]{t^{N-1}}+O\left(\sqrt[N]{t^{N-1}}\right)$ при $t \rightarrow 0$, то неравенства

$$
f_{2}(0, t)<\sqrt[N]{t}<f_{1}(0, t)
$$

выполняются для достаточно малых $t>0$. Найдем представления для остальных сингулярных решений уравнения (3), которые появляются при $t>0$. Пусть существуют $z_{0} \in \mathbb{H}$ и $t_{0}>0$ такие, что $f\left(z_{0}, t_{0}\right)=\sqrt[N]{t_{0}}$. Тогда $\left(f\left(z_{0}, t_{0}\right), \sqrt[N]{t_{0}}\right)$ является сингулярной точкой уравнения (3) и сингулярное решение $f\left(z_{0}, t\right)$ разлагается в ряд по степеням $\left(t-t_{0}\right)^{l / m}, m \in \mathbb{N}$,

$$
f\left(z_{0}, t\right)=\sqrt[N]{t_{0}}+\sum_{l=1}^{\infty} b_{l / m}\left(t-t_{0}\right)^{l / m} .
$$

Подставляем (15) в (3) и получаем:

$$
\begin{gathered}
\sum_{l=1}^{\infty} \frac{l b_{l / m}\left(t-t_{0}\right)^{l / m-1}}{m} \times \\
\times\left(\sum_{l=1}^{\infty} b_{l / m}\left(t-t_{0}\right)^{l / m}-\sum_{l=1}^{\infty} \frac{(-1)^{l-1}(N-1)(2 N-1) \ldots((l-1) N-1)\left(t-t_{0}\right)^{l}}{N^{l} l ! t_{0}^{l-1 / N}}\right)=2 .
\end{gathered}
$$

Приравнивая коэффициенты при одинаковых степенях в обеих частях уравнения (16), замечаем, что $b_{l / m} \neq 0$ только в случае $m=2$, при этом

$$
\left(b_{1 / 2}\right)^{2}=4 .
$$

Это уравнение предлагает два возможных значения $b_{1 / 2}=2$ и $b_{1 / 2}=-2$ для двух ветвей $f_{1}\left(z_{0}, t\right)$ и $f_{2}\left(z_{0}, t\right)$ сингулярного решения (15). Выберем, например, значение $b_{1 / 2}=2$ и получим рекуррентную формулу для коэффициентов ветви $f_{1}\left(z_{0}, t\right)$,

$$
b_{1 / 2}=2, \quad b_{l / 2}=\frac{1}{l+1}\left(c_{n / 2}-\frac{1}{2} \sum_{k=2}^{l-1} k b_{k / 2}\left(b_{(l-k+1) / 2}-c_{(l-k+1) / 2}\right)\right), \quad l \geq 2,
$$

где

$$
c_{(2 k-1) / 2}=0, \quad c_{k}=\frac{(-1)^{k-1}(N-1)(2 N-1) \ldots((k-1) N-1)}{N^{k} k ! t_{0}^{k-1 / N}}, \quad k=1,2, \ldots .
$$

Так как

$$
\begin{gathered}
f_{1}\left(z_{0}, t\right)=\sqrt[N]{t_{0}}+2 \sqrt{t-t_{0}}+O\left(\sqrt{t-t_{0}}\right), \\
f_{2}\left(z_{0}, t\right)=\sqrt[N]{t_{0}}-2 \sqrt{t-t_{0}}+O\left(\sqrt{t-t_{0}}\right), \\
\sqrt[N]{t}=\sqrt[N]{t_{0}}+\frac{1}{N t_{0}^{1-1 / N}}\left(t-t_{0}\right)+O\left(t-t_{0}\right), \quad t \rightarrow t_{0}+0,
\end{gathered}
$$

то неравенства $f_{2}\left(z_{0}, t\right)<\sqrt[N]{t}<f_{1}\left(z_{0}, t\right)$ выполняются для всех $t>t_{0}$, близких к $t_{0}$. 


\section{2. ВСПОМОГАТЕЛЬНЫЕ РЕЗУЛЬТАТЫ}

Из теории дифференциальных уравнений следует, что различные интегральные кривые уравнения (3) пересекаются только в сингулярной точке $(0,0)$ (см. [10, с. 138]).

Лемма 3. При $0<t_{0}<t$ для достаточно малых $t>0$ выполняются следующие неравенства:

$$
f_{2}(0, t)<f_{2}\left(z_{0}, t\right)<\sqrt[N]{t}<f_{1}\left(z_{0}, t\right)<f_{1}(0, t)
$$

где $\left(f\left(z_{0}, t_{0}\right), \sqrt[N]{t_{0}}\right)-$ сингулярная точка уравнения (3).

Доказательство. Достаточно доказать первое и последнее неравенства леммы 3. Покажем, что $f_{1}\left(z_{0}, t\right)<f_{1}(0, t)$. Вычитая одно из другого уравнения

$$
\begin{array}{cc}
\frac{d f_{1}(0, t)}{d t}=\frac{2}{f_{1}(0, t)-\sqrt[N]{t}}, & f_{1}(0,0)=0, \\
\frac{d f_{1}\left(z_{0}, t\right)}{d t}=\frac{2}{f_{1}\left(z_{0}, t\right)-\sqrt[N]{t}}, & f_{1}\left(z_{0}, t_{0}\right)=\sqrt[N]{t_{0}},
\end{array}
$$

получаем

$$
\frac{d\left(f_{1}(0, t)-f_{1}\left(z_{0}, t\right)\right)}{d t}=\frac{2\left(f_{1}\left(z_{0}, t\right)-f_{1}(0, t)\right)}{\left(f_{1}(0, t)-\sqrt[N]{t}\right)\left(f_{1}\left(z_{0}, t\right)-\sqrt[N]{t}\right)}
$$

Перепишем последнее равенство в виде

$$
\frac{d \log \left(f_{1}(0, t)-f_{1}\left(z_{0}, t\right)\right)}{d t}=-\frac{2}{\left(f_{1}(0, t)-\sqrt[N]{t}\right)\left(f_{1}\left(z_{0}, t\right)-\sqrt[N]{t}\right)} .
$$

Предположим, что существует наименьшее число $T>t_{0}$, для которого выполняется равенство $f_{1}(0, T)=f_{1}\left(z_{0}, T\right)$. Отсюда следует, что

$$
\int_{t_{0}}^{T} \frac{d t}{\left(f_{1}(0, t)-\sqrt[N]{t}\right)\left(f_{1}\left(z_{0}, t\right)-\sqrt[N]{t}\right)}=\infty
$$

Вычисляя $a_{2 N-3}^{+}=-(N-1)(2 N)^{2}$, запишем асимптотическое разложение:

$$
f_{1}(0, t)=\sqrt[N]{t}+2 N \sqrt[N]{t^{N-1}}-(N-1)(2 N) \sqrt[N]{t^{2 N-3}}+O\left(\sqrt[N]{t^{2 N-3}}\right), \quad t \rightarrow+0
$$

Существует $T^{\prime}>0$ такое, что для $0<t<T^{\prime}$ выполняется неравенство $\sqrt[N]{t}+2 N \sqrt[N]{t^{N-1}}>f_{1}(0, t)$. Для оценки интеграла в левой части равенства (19) необходимо изучить поведение функции $f_{1}\left(z_{0}, t\right)-\sqrt[N]{t}$ с использованием дифференциального уравнения:

$$
\frac{d\left(f_{1}\left(z_{0}, t\right)-\sqrt[N]{t}\right)}{d t}=\frac{2}{f_{1}\left(z_{0}, t\right)-\sqrt[N]{t}}-\frac{1}{N \sqrt[N]{t^{N-1}}}=\frac{2 N \sqrt[N]{t^{N-1}}-f_{1}\left(z_{0}, t\right)+\sqrt[N]{t}}{N \sqrt[N]{t^{N-1}}\left(f_{1}\left(z_{0}, t\right)-\sqrt[N]{t}\right)}
$$

Так как

$$
f_{1}\left(z_{0}, t\right)-\sqrt[N]{t}=2 \sqrt[N]{t-t_{0}}+O\left(\sqrt[N]{t-t_{0}}\right)
$$

то правая часть (20) положительна при $0<t<T^{\prime}, f_{1}\left(z_{0}, t\right)-\sqrt[N]{t}$ растет вместе с $t, t_{0}<t<T<T^{\prime}$, и интеграл в левой части (19) конечен. Полученное противоречие с равенством (19) отрицает существование $T$ с заданными свойствами и доказывает последнее неравенство в лемме 3.

Доказательство того, что $f_{2}(0, t)<f_{2}\left(z_{0}, t\right)$, проводится аналогично.

Лемма 4. При $0<t_{1}<t_{0}<t$ для достаточно малых $t>0$ выполняются неравенства

$$
f_{2}\left(z_{1}, t\right)<f_{2}\left(z_{0}, t\right), \quad f_{1}\left(z_{0}, t\right)<f_{1}\left(z_{1}, t\right),
$$

где $\left(f\left(z_{1}, t_{1}\right), \sqrt[N]{t_{1}}\right)$ и $\left(f\left(z_{0}, t_{0}\right), \sqrt[N]{t_{0}}\right)$ сингулярные точки уравнения (3).

Доказательство. Доказательство леммы 4 аналогично проведенному в лемме 3 с заменой 0 на $z_{1}$. 


\section{3. СВОЙСТВА РЕШЕНИЙ УРАВНЕНИЯ ЛЕВНЕРА}

Решение $f(z, t)$ дифференциального уравнения Левнера (3) аналитично по $z \in \mathbb{H} \backslash K_{t}$ для фиксированного $t \geq 0$, дифференцируемо по $t \in\left(0, t_{0}\right), t_{0}>0$, и непрерывно на $\left[0, t_{0}\right]$ для фиксированного $z \in \mathbb{H} \backslash K_{t_{0}}$. Функция $f(\cdot, t)$ допускает продолжение на замыкание области $\mathbb{H} \backslash K_{t}$, которое непрерывно в определенном смысле и устанавливает взаимно однозначное соответствие между простыми концами области $\mathbb{H} \backslash K_{t}$ и точками $\mathbb{R}$. Если простой конец области $\mathbb{H} \backslash K_{t_{0}}$, соответствующий точке $\sqrt[N]{t_{0}}$, содержит точку $z_{0}\left(t_{0}\right), t_{0}>0$, то $\left(f\left(z_{0}\left(t_{0}\right), \sqrt[N]{t_{0}}\right)\right.$ является сингулярной точкой для уравнения (3).

Зафиксируем $\tau>0$ и рассмотрим функцию $\varphi_{\tau}(t):=f(z(\tau), t), 0 \leq t \leq \tau$, где $f(z(\tau), \tau)=\sqrt[N]{\tau}$, $f(z(\tau), t)$ определяется разложением (15) с $m=2, \tau=t_{0}, z(\tau):=z_{0}$, а коэффициенты $b_{l / 2}$ в (15) вычисляются по формулам (17), (18).

Предложение 1. Функция $\varphi_{\tau}(t)$ аналитична на $(0, \tau)$, непрерывна на $[0, \tau]$, определяет кривую, ортогональную вещественной оси $\mathbb{R}$ и соединяющую точки $\sqrt[N]{\tau} u z(\tau)$. Точка $z(\tau)$ определяется однозначно для всякого достаточно малого $\tau>0$.

Доказательство. Функция $f(\cdot, \tau): \mathbb{H} \backslash K_{\tau} \rightarrow \mathbb{H}$ аналитична в области $\mathbb{H} \backslash K_{\tau}$ и взаимно однозначно сопоставляет простым концам границы этой области все вещественные точки. В частности, точке $\sqrt[N]{\tau}$ сопоставляется некоторый простой конец, произвольную точку которого обозначим через $z(\tau)$. Так как точка $(f(z(\tau), \tau), \sqrt[N]{\tau})$ является сингулярной точкой для уравнения (3), то справедливо представление (15) с $m=2, b_{l / 2}=2$, из которого следует, что

$$
f(z(\tau), t)=\sqrt[N]{\tau}+2 i \sqrt{|t-\tau|}+b_{1}(t-\tau)+O(t-\tau), \quad t \rightarrow \tau-0, \quad b_{1} \in \mathbb{R} .
$$

Отсюда заключаем, что функция $f(z(\tau), t), 0 \leq t \leq \tau$ определяет кривую, ортогональную вещественной оси в точке $\sqrt[N]{\tau}$, и $f(z(\tau), t) \in \mathbb{H}$ для $0 \leq t<\tau$. Это означает, что $z(\tau) \notin K_{t}$ для всех $t \in[0, \tau)$. Поскольку $f(z(\tau), t) \neq \sqrt[N]{t}$ на $[0, \tau)$, то $\varphi_{\tau}(t)$ аналитична на $(0, \tau)$, непрерывна на $[0, \tau]$, $\varphi_{\tau}(\tau)=\sqrt[N]{\tau}, \varphi_{\tau}(0)=f(z(\tau), 0)=z(\tau)$.

Осталось показать, что точка $z(\tau)$ определяется однозначно. Действительно, ряд по степеням $\sqrt{t-\tau}$ в представлении (15) с $m=2, t_{0}=\tau$, сходится в некотором круге радиуса $R_{0}>0$, и коэффициенты ряда не зависят от выбора точки $z_{0}=z(\tau)$, содержащейся в простом конце, соответствующем точке $\sqrt[N]{\tau}$. Функция $\varphi_{\tau}(t)$ допускает аналитическое продолжение вдоль интервала $(0, \tau)$, которое определяется только начальным элементом (15) и не зависит от выбора $z(\tau)$. Следовательно, значение $\varphi_{\tau}(0)=z(\tau)$ определяется однозначно, что завершает доказательство предложения 1 .

Доказательство теоремы 1. Для $\tau>0$ функция $f(\cdot, \tau)$ конформно отображает область $\mathbb{H} \backslash K_{\tau}$ на $\mathbb{H}$. Множество $K_{\tau} \subset \mathbb{H}$ порождается управляющей функцией $\sqrt[N]{t}$. Продолженная функция $f(\cdot, \tau)$ взаимно однозначно отображает множество простых концов области $\mathbb{H} \backslash K_{\tau}$ на $\mathbb{R}$. Один из простых концов, содержащий точку $z(\tau)$, отображается функцией $f(z(\tau), \tau)$ на $\sqrt[N]{\tau}$.

Леммы 3 и 4 описывают структуру прообраза $\mathbb{H}$ при отображении $f\left(\cdot, \tau_{0}\right)$. Все сингулярные решения $f_{1}(0, t), f_{2}(0, t), f_{1}(z(\tau), t), f_{2}(z(\tau), t), 0<\tau<t<T$, уравнения (3) являются вещественными и удовлетворяют леммам 3 и 4 . Сегмент $I=\left[f_{2}(0, t), f_{1}(0, t)\right] \subset \mathbb{R}$ объединяет сегменты $I_{2}=\left[f_{2}(0, t), \sqrt[N]{t}\right]$ и $I_{1}=\left[\sqrt[N]{t}, f_{1}(0, t)\right]$. Сегмент $I_{2}$ состоит из точек $f_{2}(z(\tau), t), 0 \leq \tau \leq t$, а сегмент $I_{1}$ состоит из точек $f_{1}(z(\tau), t), 0 \leq \tau \leq t$. Значит, все точки $z(\tau), 0 \leq \tau \leq t$, принадлежат границе $\partial\left(\mathbb{H} \backslash K_{t}\right)$ области $\mathbb{H} \backslash K_{t}$. Согласно предложению 1 простой конец области $\mathbb{H} \backslash K_{t}$, содержащий $z(\tau), 0<\tau<t$, состоит из единственной точки $z(\tau)$, которая должна быть достижимой граничной точкой. Причем $z(\tau)$ определяет ровно два простых конца, соответствующих функциям $f_{1}(z(\tau), t)$ и $f_{2}(z(\tau), t)$. Это доказывает, что $\{z(\tau): 0 \leq \tau \leq t\}$ представляет множество точек простой кривой $\gamma$, которая и является множеством $K_{t}$ с простыми концами, соответствующими точкам на разных сторонах кривой $\gamma$. Обратная функция $f^{-1}(w, t)$ отображает $\mathbb{H}$ на $\mathbb{H} \backslash \gamma(t)$ для достаточно малых $t>0$.

Осталось показать, что $\gamma(t)$ является $C^{1}$-кривой. Фиксируем достаточно малое $t_{0}>0$ и обозначим через $g\left(w, t_{0}\right)=f^{-1}\left(w, t_{0}\right)$ функцию, обратную к функции $f\left(z, t_{0}\right)$. Положим $h(w, t):=f\left(g\left(w, t_{0}\right), t\right)$, 
$t \geq t_{0}$. Функция $f\left(z, t_{0}\right)$ отображает $\mathbb{H} \backslash \gamma\left(t_{0}\right)$ на $\mathbb{H}, \gamma_{t_{0}}=\left\{\gamma(\tau), 0 \leq \tau \leq t_{0}\right\}, f\left(\gamma\left(t_{0}\right), t_{0}\right)=\sqrt[N]{t_{0}}$. При этом отображении кривая $\gamma\left[t_{0}, t\right]:=\left\{\gamma(\tau), t_{0} \leq \tau \leq t\right\}$ переходит в кривую $\gamma^{*}\left[t_{0}, t\right]:=f\left(\gamma\left[t_{0}, t\right], t_{0}\right)$, $\gamma^{*}\left(t_{0}\right)=\sqrt[N]{t_{0}}$. Поэтому функция $h(w, t)$ отображает $\mathbb{H} \backslash \gamma^{*}\left[t_{0}, t\right]$ на $\mathbb{H}$. Запишем разложение $h(w, t)$ в окрестности бесконечно удаленной точки:

$$
h(w, t)=g\left(w, t_{0}\right)+\frac{2 t}{g\left(w, t_{0}\right)}+O\left(\frac{1}{g^{2}\left(w, t_{0}\right)}\right)=w+\frac{2\left(t-t_{0}\right)}{w}+O\left(\frac{1}{w^{2}}\right) .
$$

После замены переменных $t_{1}:=t-t_{0}, h_{1}\left(w, t_{1}\right):=h\left(w, t_{0}+t_{1}\right)$ функция $h_{1}$ имеет разложение (1) и удовлетворяет дифференциальному уравнению:

$$
\frac{d h_{1}\left(w, t_{1}\right)}{d t_{1}}=\frac{2}{h_{1}\left(w, t_{1}\right)-\sqrt[N]{t_{1}+t_{0}}}, \quad h_{1}(w, 0)=w, \quad w \in \mathbb{H} .
$$

Управляющая функция $\lambda\left(t_{1}\right)=\sqrt[N]{t_{1}+t_{0}}$ в $(21)$ является аналитической при $t_{1} \geq 0$. В классической версии уравнения Левнера для отображений круга в круг известно [3, с. 59], что гладкая управляющая функция генерирует отображения круга на круг с гладким разрезом. Этот результат без особого труда переносится на уравнение Левнера (2) и на его частный случай (21) (см. например [8] и приведенные там ссылки). Таким образом, кривая $\gamma^{*}\left[t_{0}, t\right]$, а вместе с ней и кривая $\gamma\left(\left[t_{0}, t_{1}\right]\right)=g\left(\gamma^{*}\left[t_{0}, t\right], t_{0}\right)$, являются $C^{1}$-кривыми. Устремляя $t_{0}$ к 0 , убеждаемся, что $\gamma_{t}=\{\gamma(\tau): 0 \leq \tau \leq t\}$ является $C^{1}$-кривой, за исключением, быть может, точки $\gamma(0)=0$, что завершает доказательство теоремы 1.

\section{4. ГАРМОНИЧЕСКИЕ МЕРЫ СТОРОН РАЗРЕЗА}

Функция $f(z, t)$, являющаяся решением уравнения (3), отображает область $\mathbb{H} \backslash \gamma(t)$ на $\mathbb{H}$. Точки двух сторон разреза $\gamma(t)$ считаются различными граничными точками области. Обозначим через $\gamma_{1}=\gamma_{1}(t)$ ту сторону $\gamma$, которая отображается продолженной функцией $f(z, t)$ на сегмент $I_{1}=\left[\sqrt[N]{t}, f_{1}(0, t)\right]$, а через $\gamma_{2}=\gamma_{2}(t)-$ сторону $\gamma$, которая является прообразом $I_{2}=\left[f_{2}(0, t), \sqrt[N]{t}\right]$ при отображении функцией $f(z, t)$.

Напомним, что гармонические меры $\omega\left(f^{-1}(i, t) ; \gamma_{k}, \mathbb{H} \backslash \gamma(t), t\right)$ дуг $\gamma_{k}$ в точке $f^{-1}(i, t)$ относительно области $\mathbb{H} \backslash \gamma(t)$ определяются функциями $\omega_{k}$, которые являются гармоническими в области $\mathbb{H} \backslash \gamma(t)$ и непрерывно продолжаются на ее замыкание, за исключением концевых точек кривой $\gamma,\left.\omega_{k}\right|_{\gamma_{k}(t)}=1$, $\left.\omega_{k}\right|_{\mathbb{R} \cup\left(\gamma(t) \backslash \gamma_{k}(t)\right)}=0, k=1,2$ (см. [16, с. 132]). Обозначим:

$$
m_{k}(t):=\omega\left(f^{-1}(i, t) ; \gamma_{k}, \mathbb{H} \backslash \gamma(t), t\right), \quad k=1,2
$$

Теорема 2. Пусть функция $f(z, t)$ является решением уравнения Левнера (3). Тогда справедливо асимптотическое соотношение:

$$
\lim _{t \rightarrow+0} \frac{m_{1}(t)}{m_{2}^{N-1}(t)}=2 N \pi^{N-2} .
$$

Доказательство. Гармоническая мера инвариантна относительно конформных преобразований. Поэтому гармонические меры

$$
\omega\left(f^{-1}(i, t) ; \gamma_{k}, \mathbb{H} \backslash \gamma(t), t\right)=\Omega\left(i ; f\left(\gamma_{k}, t\right), \mathbb{H}, t\right)
$$

определяются функциями $\Omega_{k}$, которые гармоничны на $\mathbb{H}$ и непрерывно продолжаются на $\mathbb{R}$, за исключением концевых точек образов $f\left(\gamma_{k}, t\right),\left.\Omega_{k}\right|_{f\left(\gamma_{k}, t\right)}=1,\left.\Omega_{k}\right|_{\mathbb{R} \backslash f\left(\gamma_{k}, t\right)}=0, k=1,2$. Решение этой задачи известно (см. [17, с. 334]). Именно

$$
m_{k}(t)=\frac{\alpha_{k}(t)}{\pi}
$$

где $\alpha_{k}(t)$ - угол, под которым наблюдается сегмент $I_{k}=I_{k}(t)$ из точки $w=i, k=1,2$. Осталось найти асимптотические разложения для $\alpha_{k}(t)$. 
Так как

$$
f_{1}(0, t)=\sqrt[N]{t}+2 N \sqrt[N]{t^{N-1}}+O(t), \quad f_{2}(0, t)=-\frac{2 N}{N-1} \sqrt[N]{t^{N-1}}+O(t), \quad t \rightarrow+0,
$$

то после элементарных геометрических рассуждений находим, что

$$
\begin{gathered}
\alpha_{1}(t)=\operatorname{arctg} f_{1}(0, t)-\operatorname{arctg} \sqrt[N]{t}=2 N \sqrt[N]{t^{N-1}}+O(t), \quad t \rightarrow+0, \\
\alpha_{2}(t)=\operatorname{arctg} \sqrt[N]{t}-\operatorname{arctg} f_{2}(0, t)=\sqrt[N]{t}+\frac{2 N}{N-1} \sqrt[N]{t^{N-1}}+O(t), \quad t \rightarrow+0 .
\end{gathered}
$$

Это означает, что

$$
\frac{m_{1}(t)}{m_{2}^{N-1}(t)}=\frac{\pi^{N-2}\left(2 N \sqrt[N]{t^{N-1}}+O(t)\right)}{\left(\sqrt[N]{t}+\frac{2 N}{N-1} \sqrt[N]{t^{N-1}}+O(t)\right)^{N-1}}=2 N \pi^{N-2}(1+O(\sqrt[N]{t})), \quad t \rightarrow+0,
$$

что приводит к (22) и завершает доказательство теоремы 2.

Замечание. Из результатов статьи [9] следует соотношение, аналогичное (22), для двух сторон разреза вдоль дуги окружности $\gamma(t)$ в $\mathbb{H}$, которая является касательной к вещественной оси в точке $z=0$.

\section{Библиографрический список}

1. Löwner $K$. Untersuchungen über schlichte konforme Abbildungen des Einheitskreises // I. Math. Ann. 1923. Vol. 89, № 1-2. P. 103-121.

2. Markina I., Vasil'ev A. Virasoro algebra and dynamics in the space of univalent functions // Contemp. Math. 2010. Vol. 525. P. 85-116.

3. Александров И. А. Параметрические продолжения в теории однолистных функций. M. : Наука, 1976. 344 с. 4. Lind J., Marshall D. E., Rohde S. Collisions and spirals of Loewner traces // Duke Math. J. 2010. Vol. 154, № 3. P. 527-573. DOI:10.1215/00127094-2010-045.

5. Куфарев П. П. Одно замечание об интегралах уравнения Лёвнера // Докл. АН СССР. 1947. Т. 57, № 7. C. 655-656.

6. Kager W., Nienhuis B., Kadanoff L. P. Exact solutions for Loewner evolutions // J. Statist. Phys. 2004. Vol. 115, № 3-4. P. 805-822.

7. Прохоров Д. В., Захаров А. М. Интегрируемость частного вида уравнения Лёвнера // Изв. Сарат. ун-та. Нов. сер. Сер. Математика. Механика. Информатика. 2010. Т. 10, вып. 2. С. 19-23.

8. Marshall D. E., Rohde S. The Loewner differential equation and slit mappings // J. Amer. Math. Soc. 2005. Vol. 18, № 4. P. 763-778.
9. Prokhorov D., Vasil'ev A. Singular and tangent slit solutions to the Löwner equation // Analysis and Mathematical Physics / eds. B. Gustafsson, A. Vasil'ev. Berlin : Birkhauser, 2009. P. 455-463.

10. Сансоне Дж. Обыкновенные дифференциальные уравнения : в 2 т. М. : Иностр. лит., 1954. Т. 2. 414 с.

11. Poincaré $H$. Sur les courbes définies par une équation différentielle // J. Math. Pures Appl. 1886. Vol. 4, № 2. P. 151-217.

12. Bendixson I. Sur les courbes définies par les équations différentielles // Acta Math. 1901. Vol. 24. P. 1-88.

13. Голубев В.В. Лекции по аналитической теории дифференциальных уравнений. М.; Л. : Гостехиздат, 1950. 398 c.

14. Сансоне Дж. Обыкновенные дифференциальные уравнения : в 2 т. М. : Иностр. лит., 1953. Т. 1.346 с.

15. Borel E. Mémoire sur les séries divergentes // Ann. Sci. École Norm. Sup. 1899. Vol. 16, № 3. P. 9-131.

16. Хейман У., Кеннеди П. Субгармонические функции. М. : Мир, 1980. 304 с.

17. Голузин Г. М. Геометрическая теория функций комплексного переменного. 2-е изд. М. : Наука, 1966. 628 c.

\section{Integrals of the Loewner Equation with Exponential Driving Function}

\section{V. Prokhorov, K. A. Samsonova}

Saratov State University, Russia, 410012, Saratov, Astrakhanskaya st., 83, ProkhorovDV@ info.sgu.ru, kris_ruzhik@mail.ru

We consider the qualitative local behavior of trajectories for the ordinary Loewner differential equation with a driving function which is inverse to the exponential function of an integer power. All the singular points and the corresponding singular solutions are described. It is shown that this driving function generates solutions to the Loewner equation which map conformally a half-plane slit along a smooth curve onto the upper half-plane. The asymptotical correspondence between harmonic measures of two slit sides is derived.

Key words: Loewner equation, harmonic measure, singular solutions, driving function, $C^{1}$-curve. 


\section{References}

1. Löwner K. Untersuchungen über schlichte konforme Abbildungen des Einheitskreises. I. Math. Ann., 1923, vol. 89, no. 1-2, pp. 103-121.

2. Markina I., Vasil'ev A. Virasoro algebra and dynamics in the space of univalent functions. Contemp. Math., 2010, vol. 525, pp. 85-116.

3. Aleksandrov I. A. Parametric continuations in the theory of univalent functions. Moscow, Nauka, 1976, 344 p. (in Russian).

4. Lind J., Marshall D. E., Rohde S. Collisions and spirals of Loewner traces. Duke Math. J., 2010, vol. 154(3), pp. 527-573. DOI: 10.1215/00127094-2010-045.

5. Kufarev P. P. Odno zamechanie ob integralakh uravneniia Levnera. [A remark on integrals of Löwner's equation] Doklady Akad. Nauk SSSR, 1947, vol. 57, no. 7, pp. 655-656 (in Russian).

6. Kager W., Nienhuis B., Kadanoff L. P. Exact solutions for Loewner evolutions. J. Statist. Phys., 2004, vol. 115, no. $3-4$, pp. $805-822$.

7. Prokhorov D. V., Zakharov A. M. Integrability of a partial case of the Löwner equation. Izv. Saratov Univ. (N. S.), Ser. Math. Mech. Inform., 2010, vol. 10, iss. 2, pp. 19-23 (in Russian).

8. Marshall D. E., Rohde S. The Loewner differential equation and slit mappings. J. Amer.Math. Soc., 2005, vol. 18 , no. 4 , pp. $763-778$.

9. Prokhorov D., Vasil'ev A. Singular and tangent slit solutions to the Löwner equation. Analysis and Mathematical Physics, eds. B. Gustafsson, A. Vasil'ev. Berlin, Birkhauser, 2009, pp. 455-463.

10. Sansone G. Equazioni differenziale nel campo reale. P. $2^{a}, 2^{a}$ ediz., Bologna, 1949.

11. Poincaré H. Sur les courbes définies par une équation différentielle. J. Math. Pures Appl., 1886, vol. 4, no. 2, pp. 151-217.

12. Bendixson I. Sur les courbes définies par les équations différentielles. Acta Math., 1901, vol. 24, pp. 1-88.

13. Golubew W. Differentialgleichungen im komplexen, veb deutsch. Berlin, Verlag Wiss., 1958, 398 p.

14. Sansone G. Equazioni differenziale nel campo reale. P. $1^{a}, 2^{a}$ ediz., Bologna, 1948.

15. Borel E. Mémoire sur les séries divergentes. Ann. Sci. École Norm. Sup., 1899, vol. 16, no. 3, pp. 9-131.

16. Hayman W., Kennedy P. Subharmonic functions. London, Academic Press, 1976.

17. Goluzin G. Geometric theory of functions of a complex variable. Transl. Math. Monographs, vol. 26, Providence, RI, AMS, 1969. 676 p.

\section{УДК 512.577}

\section{О НАСЛЕДСТВЕННОСТИ ФОРМАЦИЙ УНАРОВ}

\section{А. Л. Расстригин}

Старший преподаватель кафредры алгебры, геометрии и математического анализа, Волгоградский государственный социально-педагогический университет, rasal@fizmat.vspu.ru

Формацией называют класс алгебраических систем, замкнутый относительно гомоморфных образов и конечных подпрямых произведений. В работе показано, что любая фрормация, состоящая из не более чем счетных унаров, является наследственной.

Ключевые слова: унар, фрормация, наследственная фрормация.

\section{1. ОПРЕДЕЛЕНИЯ}

Формацией называется класс алгебраических систем, замкнутый относительно взятия гомоморфных образов и конечных подпрямых произведений. Формацию называют конечной, если она содержит лишь конечные системы. Мы будем называть формацию не более чем счетной, если она содержит лишь не более чем счетные системы.

Пусть $\mathfrak{X}-$ совокупность алгебраических систем. Через $\mathrm{H}(\mathfrak{X})$ и $\mathrm{R}_{0}(\mathfrak{X})$ обозначаются совокупности всех гомоморфных образов и конечных подпрямых произведений $\mathfrak{X}$-систем соответственно. Через $\mathrm{S}(\mathfrak{X})$ обозначается класс всех подсистем $\mathfrak{X}$-систем. Класс $\mathfrak{X}$ называется наследственныл, если $\mathrm{S}(\mathfrak{X}) \subseteq \mathfrak{X}$. Через form $\mathfrak{X}$ ( $\operatorname{sform} \mathfrak{X})$ обозначается наименьшая (наименьшая наследственная) формация, содержащая $\mathfrak{X}$ или, иначе, порожденная совокупностью $\mathfrak{X}$. Через Si $\mathfrak{X}$ обозначается совокупность всех подпрямо неразложимых $\mathfrak{X}$-систем. Множество целых неотрицательных чисел обозначается $\mathbb{N}_{0}$, $\mathbb{N}=\mathbb{N}_{0} \backslash\{0\}$ и $\mathbb{Z}-$ множество целых чисел.

(C) РасстригинА. ^., 2013 